\title{
The Mouse Brain Metabolome
}

\section{Region-Specific Signatures and Response to Excitotoxic Neuronal Injury}

\author{
Christian Jaeger, Enrico Glaab, Alessandro Michelucci, Tina M. Binz, Sandra Koeglsberger, Pierre Garcia, Jean-Pierre Trezzi, \\ Jenny Ghelfi, Rudi Balling, and Manuel Buttini
}

From the Luxembourg Centre for Systems Biomedicine, University of Luxembourg, Esch-sur-Alzette, Luxembourg

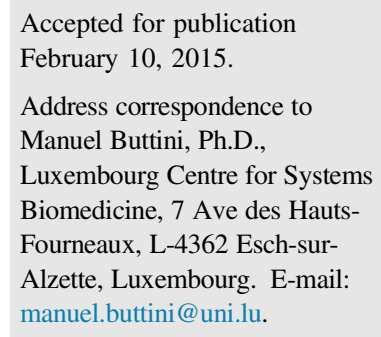

\begin{abstract}
Neurodegeneration is a multistep process characterized by a multitude of molecular entities and their interactions. Systems analyses, or omics approaches, have become an important tool in characterizing this process. Although RNA and protein profiling made their entry into this field a couple of decades ago, metabolite profiling is a more recent addition. The metabolome represents a large part or all metabolites in a tissue, and gives a snapshot of its physiology. By using gas chromatography coupled to mass spectrometry, we analyzed the metabolic profile of brain regions of the mouse, and found that each region is characterized by its own metabolic signature. We then analyzed the metabolic profile of the mouse brain after excitotoxic injury, a mechanism of neurodegeneration implicated in numerous neurological diseases. More important, we validated our findings by measuring, histologically and molecularly, actual neurodegeneration and glial response. We found that a specific global metabolic signature, best revealed by machine learning algorithms, rather than individual metabolites, was the most robust correlate of neuronal injury and the accompanying gliosis, and this signature could serve as a global biomarker for neurodegeneration. We also observed that brain lesioning induced several metabolites with neuroprotective properties. Our results deepen the understanding of metabolic changes accompanying neurodegeneration in disease models, and could help rapidly evaluate these changes in preclinical drug studies. (Am J Pathol 2015, 185: 1699-1712; http://dx.doi.org/10.1016/j.ajpath.2015.02.016)
\end{abstract}

Neurodegeneration, or the pathological loss of neuronal structure and function that culminates in neuron death, is typically assessed by histological and/or biochemical measurements of specific markers or mediators associated with neuronal demise and with the accompanying reaction of glial cells.

During the past decades, to better understand the multiple features of neurodegeneration, systems, or omics, approaches have made their entry into the field. Transcriptomics, in particular, have been used extensively to characterize, at the gene expression level, region-specific profiles $^{1}$ and degenerative processes in neurons affected in different diseases. ${ }^{2,3}$ More recently, metabolomics, or the comprehensive study of metabolites, has been added to the omics toolbox to study the brain. ${ }^{4}$ The metabolome represents the collection of all or part of the molecules (metabolic intermediates, signaling molecules, and secondary metabolites) that are the product of biochemical processes in a specimen. Metabolic profiling gives an instantaneous snapshot of the physiology of that specimen. The platforms used for such analyses include nuclear magnetic resonance spectroscopy and mass spectrometry-based approaches. ${ }^{5-7}$

Many metabolome studies on the brain and its diseases have been focusing on profiling on human's or rodent model's cerebrospinal fluid, in an effort to find biomarkers

\footnotetext{
Supported by internal funding from the Luxembourg Centre for Systems Biomedicine, University of Luxembourg.

C.J., E.G., and A.M. contributed equally to this work.

Disclosures: None declared.

Current address of T.M.B., Zurich Institute of Forensic Medicine, University of Zurich, Zurich, Switzerland.
} 
of disease severity and progression. ${ }^{4,8}$ Fewer are looking at the actual brain tissues. An integrative study mapping the metabolome of different brain regions, and the metabolic response to injury, with an eye on associations such changes have with neuropathological and molecular markers of neurodegeneration, is still lacking. Such studies are timely in view of the rapidly expanding populations of genetic rodent models for neurological diseases and their use in drug efficacy studies.

Therefore, we used gas chromatography coupled to mass spectrometry (GC-MS), which allows us to detect a large population of metabolites, to map the metabolome of different mouse brain regions. The various neuronal populations that populate the different regions of the mammalian brain and that have varying responses and vulnerabilities to different diseases have not yet been characterized at this level. We then used that approach to detect metabolic changes in response to neurodegeneration induced by excitotoxic challenge, and compared these changes to pathological and molecular alterations that reflect neuronal injury and gliosis.

We found that, at baseline, each mouse brain region has its particular metabolic signature that could be distinguished from that of other regions via principal component analysis or, to a better extent, by supervised machine learning algorithms. We also found that, after excitotoxic injury, the metabolic signature of the cortex, a brain region that is severely affected by this process, shifts to a unique metabolic signature in affected animals. To validate these bioinformatical observations, we compared excitotoxicity-induced metabolic changes with histopathological and molecular measures of neurodegeneration and gliosis. Our results showed that a specific metabolite signature, revealed by machine learning, rather than individual metabolites, was the most robust correlate of neurodegeneration. Our data support the notion that metabolic profiling of brain regions is a valuable addition to the study of central nervous system (CNS) in health and disease, and pave the way for linking metabolite changes to specific molecular CNS disease markers.

\section{Materials and Methods}

\section{Animals}

All animal experimentation was approved by the university and the appropriate Luxembourg governmental agencies (Ministry of Health and Ministry of Agriculture).

Mice (aged 4 to 6 months) were of the FVB/N strain and purchased from Charles River (Bois-des-Oncins, France). They were housed 1 to 2 weeks under a 12-hour light/dark cycle with ad libitum access to water and food until euthanasia, or treatment followed by euthanasia, in a conventional animal housing facility. For treatment, mice were randomly assigned to the kainic acid (KA) groups or the control group. Mice were injected peritoneally with $20 \mathrm{mg} /$ $\mathrm{kg}$ KA dissolved in sterile phosphate-buffered saline (PBS) or with PBS (vehicle control). Mice were euthanized either 2 or 7 days after injection.

At euthanasia, mice were deeply anesthetized with a ketamine-medetomidine mix (100 and $1 \mathrm{mg} / \mathrm{kg}$, respectively), then transcardially perfused with PBS to remove the blood. The brains were quickly removed, and one hemibrain was drop fixed in ice-cold phosphate-buffered $4 \%$ paraformaldehyde to be processed for histology. The other hemibrain was dissected within 1 minute using ultrafine forceps on an ice-cold $\left(4^{\circ} \mathrm{C}\right)$ plate into the following brain regions: cortex, hippocampus, striatum, midbrain, cerebellum, and brainstem. The dissected parts were quickly weighed, then snap frozen on crushed dry ice, and stored at $-80^{\circ} \mathrm{C}$ until use.

\section{Metabolite Extraction and Measurement in Brain Tissues by Nontargeted Metabolite Analysis}

For the analysis of metabolites of mouse brain regions at baseline, different dissected brain regions (see above) of a total of six mice (3 males and 3 females) were used.

For the analysis of metabolites of mouse brain cortex after excitotoxic injury, the cortices of 14 vehicle-treated and of 14 KA-treated mice ( 6 females and 8 males in each group) were used. The cortex was used because it is one of the regions rich in receptors targeted by $\mathrm{KA},{ }^{9}$ and because of its relatively large size, the yield of sufficient material for this study was ensured.

On the day of the extraction, three 7-mm grinding balls were added to each tissue frozen sample into the Eppendorf (Hamburg, Germany) tube. The sample tubes were then put in liquid nitrogen for 15 seconds. At the same time, a grinding block was cooled in liquid nitrogen for 15 seconds. Sample tubes were then put into the grinding block and pulverized in the ball mill (Retsch, Aartselaar, Belgium) for 2 minutes at $20 \mathrm{Hertz}$ per second (s-1) to yield a fine powder. For homogenization, five small grinding balls $(1 \mathrm{~mm})$ and the appropriate amount of extraction fluid (methanol/distilled water, 40:8.5 v/v) were added to the pulverized samples ( $485 \mu \mathrm{L} / 100 \mathrm{mg}$ tissue) and milled for 2 minutes at $20 \mathrm{~s}-1$, leading to a homogeneous fluid.

A liquid-liquid extraction method was used for metabolite extraction from each brain tissue sample. First chloroform (per $100 \mathrm{mg}$ tissue) was added to the homogenized tissue fluid, then distilled water $(200 \mu \mathrm{L} / 100 \mathrm{mg}$ tissue). The mixture was incubated on a Thermomixer (Eppendorf) for 20 minutes at $1300 \mathrm{rpm}$ at $4^{\circ} \mathrm{C}$. After the incubation period, the suspension was centrifuged for 5 minutes at $5000 \times g$ at $4^{\circ} \mathrm{C}$, and $30 \mu \mathrm{L}$ of the upper aqueous phase (containing polar metabolites) was transferred into a sample vial with a microinsert for speed vacuum evaporation at $-4^{\circ} \mathrm{C}$ using a refrigerated CentriVap Concentrator (Labconco, Kansas City, MO). The resulting dried samples were used for GC-MS analysis.

\section{Metabolite Measurements by GC-MS}

Metabolite derivatization was performed using a Gerstel multiple purpose sampler (Muehlheim-an-der-Ruhr, Germany). 
Dried polar metabolites were dissolved in $15 \mu \mathrm{L}$ pyridine, containing $20 \mathrm{mg} / \mathrm{mL}$ methoxyamine hydrochloride, at $40^{\circ} \mathrm{C}$ for 30 minutes under shaking. After adding $15 \mu \mathrm{L} \mathrm{N}$-methyl- $N$ trimethylsilyl-trifluoroacetamide, samples were incubated at $40^{\circ} \mathrm{C}$ for 30 minutes under continuous shaking.

GC-MS analysis was performed by using an Agilent 7890A GC coupled to an Agilent 5975C inert XL MSD (Diegem, Belgium). A sample volume of $1 \mu \mathrm{L}$ was injected into a Split/ Splitless inlet in splitless mode at $270^{\circ} \mathrm{C}$. The gas chromatograph was equipped with a 30-m Agilent J\&W GC (DB-35MS) capillary column +5 -m DuraGuard capillary preceding the analytical column. Helium was used as carrier gas with a constant flow rate of $1 \mathrm{~mL}$ per minute. For the analysis of brain regions, the $\mathrm{GC}$ oven temperature was held at $80^{\circ} \mathrm{C}$ for 1 minute and increased to $300^{\circ} \mathrm{C}$ at $36^{\circ} \mathrm{C}$ per minute. After 10 minutes, the temperature was increased by $10^{\circ} \mathrm{C}$ per minute to $325^{\circ} \mathrm{C}$, then held at that temperature for 4 minutes. The total run time for each sample was 59.167 minutes. After some in-house protocol simplifications, the procedure for the KA-treated brains and their vehicle controls was as follows: the GC oven temperature was held at $80^{\circ} \mathrm{C}$ for 1 minute and increased to $260^{\circ} \mathrm{C}$ at $36^{\circ} \mathrm{C}$ per minute. After 1 minute, the temperature was increased at $22^{\circ} \mathrm{C}$ per minute, followed by an additional constant temperature period at $325^{\circ} \mathrm{C}$ for 2 minutes. The transfer line temperature was set constantly to $280^{\circ} \mathrm{C}$. The MSD was operating under electron ionization at $70 \mathrm{eV}$. The MS source was held at $230^{\circ} \mathrm{C}$ and the quadrupole at $150^{\circ} \mathrm{C}$. Full-scan mass spectra were acquired from $\mathrm{m} / \mathrm{z} 70$ to 800 . The total run time of one sample was 11.955 minutes.

All GC-MS chromatograms were processed using MetaboliteDetector ${ }^{10}$ for nontargeted analysis. The software package supports automatic deconvolution of all mass spectra and calculates the retention indices on the basis of a retention index marker. The obtained mass spectra were matched against an in-house mass spectral library. Compounds were annotated by retention index and mass spectrum. Selected fragment ions unique for each individual metabolite were used for quantification, and, finally, each compound was normalized by the summed sample signal measured for each individual samples. This approach, named total ion current normalization, has turned out to be a reliable way of comparing multiple samples and correct for possible variation in sample preparation and instrument drift. ${ }^{11,12}$ A total of 332 polar metabolites were measured, of which 29\% (95 metabolites) were identified.

\section{RNA Isolation and RT-PCR}

To assess the molecular profile of reactive microglia after excitotoxic injury, transcripts for IL-1 $\beta$ (Illb) and chemokine (C-C motif) ligand $2(\mathrm{Ccl} 2)$ were measured on extracted RNA. ${ }^{13}$ To assess the molecular profile of reactive astroglia after excitotoxic injury, transcripts for tissue inhibitor of metalloproteinase 1 (Timp1), vimentin (Vim), and serine peptidase inhibitor, clade A, member 3N (Serpina3n, Serp3n) were measured on extracted RNA. ${ }^{14}$

For RNA isolation, the interphase resulting from the methanol-chloroform extraction (see above) was used. Previous studies have shown that RNA obtained with this protocol was of high quality and suitable for transcriptlevel measurements. ${ }^{15,16}$ Total RNA was purified using the Qiagen RNeasy Mini Kit (Qiagen, Venlo, the Netherlands), as per the manufacturer's instructions. First-strand cDNA was synthesized from $0.5 \mu \mathrm{g}$ of total RNA using Superscript III (Invitrogen, Gent, Belgium) with $1 \mu \mathrm{L}(50 \mu \mathrm{mol} / \mathrm{L})$ per reaction oligo $(\mathrm{dT})_{20}$ as primer. Individual $20 \mu \mathrm{L}$ SYBR Green real-time PCRs consisted of $2 \mu \mathrm{L}$ of diluted cDNA, $10 \mu \mathrm{L}$ of $2 \times$ iQ SYBR Green Supermix (Bio-Rad, Temse, Belgium), and $0.5 \mu \mathrm{L}$ of each $10 \mu \mathrm{mol} / \mathrm{L}$ optimized forward and reverse primers in $7 \mu \mathrm{L}$ RNase-free water. Ribosomal protein L27 (Rpl27), Illb, and Ccl2 primer sequences were designed using Beacon Designer software version 5.10 (Bio-Rad) and were provided by Eurogentec (Searing, Belgium). Timp1, Vim, and Serp3n primer sequences were designed using the National Center for Biotechnology Information Database/Primer-BLAST tool (http://www. ncbi.nlm.nih.gov/tools/primer-blast) and were provided by Eurogentec. All primer sequences are shown in Table 1.

The PCR was performed on a Light Cycler 480 (Roche Diagnostics, Rotkreuz, Switzerland), using a three-stage program provided by the manufacturer: 10 minutes at $95^{\circ} \mathrm{C}$ and 40 cycles of 30 seconds at $95^{\circ} \mathrm{C}, 30$ seconds at $60^{\circ} \mathrm{C}$, and 30 seconds at $72^{\circ} \mathrm{C}$, followed by 10 seconds at $70^{\circ} \mathrm{C}$ to $95^{\circ} \mathrm{C}$ melting curves. All experiments included two notemplate controls and were performed on the number of mice mentioned in the previous section with two technical replicates for each sample. For normalization, ribosomal protein L27 ( $R p l 27$ ) was amplified simultaneously.

The threshold cycle of each gene was determined as PCR cycles at which an increase in reporter fluorescence above a

Table 1 RT-PCR Primer Sequences for Molecular Markers of Microglia and Astroglia

\begin{tabular}{|c|c|c|}
\hline Primer name & Forward primer & Reverse primer \\
\hline $\mathrm{Il}-1 \beta$ & 5'-GGTTCTGTCCCTTTCACTCAC-3' & $5^{\prime}-\mathrm{TGCCTCTTCTGCCAGTTCC-3^{ \prime }}$ \\
\hline Ccl2 & 5'-CACTCACCTGCTGCTACTCATTC-3' & 5'-GCTTCTTTGGGACACCTGCTG-3' \\
\hline Timp1 & 5'-CCAGAGCCGTCACTTTGCTT-3' & $5^{\prime}$-AGGAAAAGTAGACAGTGTTCAGGCTT-3' \\
\hline Vim & $5^{\prime}-$ TCCAGAGAGAGGAAGCCGAA- $3^{\prime}$ & 5'-GCAAGGATTCCACTTTCCGTT-3' \\
\hline Serpina3n & $5^{\prime}-\mathrm{TATCTGCCTCCACCCAAAAG-3^{ \prime }}$ & $5^{\prime}$-GCCAGATGTGGACAAAGTGA-3' \\
\hline Rpl27 & $5^{\prime}$-ACATTGACGATGGCACCTC-3' & 5'-GCTTGGCGATCTTCTTCTTG-3' \\
\hline
\end{tabular}

Ccl2, chemokine (C-C motif) ligand 2; Rpl27, ribosomal protein L27; Serpina3n, serine peptidase inhibitor, clade A, member 3N; Timp1, tissue metallopeptidase inhibitor 1 ; Vim, vimentin. 
baseline signal was measured. The difference in threshold cycles between the target gene and reference gene Rpl27 yielded the standardized expression level $\left(\mathrm{dC}_{\mathrm{T}}\right)$. The expression level of each gene was calculated with the formula $2^{-\mathrm{dC}_{\mathrm{T}}}$.

Generation of Tissue Sections, Immunohistochemistry, and Quantitation of Neurodegeneration and Gliosis

Hemibrains were left in fixative (phosphate-buffered $4 \%$ paraformaldehyde) at $4^{\circ} \mathrm{C}$ for 48 hours. They were then transferred to PBS containing $0.02 \%$ sodium azide as preservative and stored at $4{ }^{\circ} \mathrm{C}$ until cutting. Free-floating sections (40 $\mu \mathrm{m}$ thick) were generated with a Leica VT1000 vibratome (Wetzlar, Germany), and stored in antifreeze medium ( $1 \% \mathrm{w} / \mathrm{v}$ polyvinyl pyrrolidone in a $1: 1 \mathrm{v} / \mathrm{v}$ PBS/ ethylene glycol mix) at $-20^{\circ} \mathrm{C}$ until immunostaining.

The following antibodies were used: anti-microtubuleassociated protein 2 (MAP2; diluted 1:800; Millipore, Overijse, Belgium), anti-synaptophysin (SYN; clone SY38, diluted 1:900; Dakopatts, Heverle, Belgium), anti-glial fibrillary acid protein (GFAP; diluted 1:4000; Dakopatts), and anti-ionizing calcium-binding adaptor molecule 1 (diluted 1:3000; Wako, Neuss, Germany).

For the detection of neurodegeneration, fluorescence immunostaining on free-floating sections was performed on the basis of a standard procedure. ${ }^{17}$ Detection of anti-MAP2 and anti-SYN was done with 1:2000 diluted Alexa488-coupled secondary antibody (Millipore) and with 1:2000 diluted Alexa-594-coupled secondary antibody (Millipore), respectively. Two sections per mouse were stained for each marker, for a total of 14 vehicle-treated mice (6 females and 8 males) and 14 KA-treated mice (6 females and 8 males). MAP2 and SYN were imaged and analyzed mainly as described. ${ }^{17}$

Briefly, MAP2-stained sections were viewed under a Zeiss Apotome 2.0 (Zaventem, Belgium) connected to a Zeiss AxioImager Z1 microscope, and digital images $(220 \times 170 \mu \mathrm{m})$, three for the cortical area of each section, were collected with a Zeiss Axiocam MRm3 camera, using a $40 \times$ objective. SYN-stained sections were viewed by a Zeiss LSM 710 laser-scanning confocal microscope, using a $20 \times$ objective and a zoom factor of 1.7 ; three to four images $(180 \times 180 \mu \mathrm{m})$ were collected from the cortical region of each section. Images were then transferred to a laptop personal computer, and percentage image area occupied by MAP2-positive dendrites or SYN-positive presynaptic terminals was quantified using the ImageJ software version 1.45 (NIH, Bethesda, MD). For each marker, values of individual animals were averaged. The analysis was performed blinded (M.B.), and codes were broken once individual animal values had been obtained.

For the detection of microgliosis and astrogliosis, immunoperoxidase staining on free-floating sections was performed using standard procedures. ${ }^{18}$ Three sections per mouse were stained for each marker, for a total of 14 vehicle-treated mice
( 6 females and 8 males) and 14 KA-treated mice ( 6 females and 8 males). The microglial marker anti-ionizing calciumbinding adaptor molecule was used at 1:3000 dilution, and the astroglial marker anti-GFAP at 1:4000 dilution. Biotinylated goat anti-rabbit (Vector, Burlingame, CA) secondary antibody was used at 1:200 dilution. The Vector Elite kit was used to visualize the signals, following manufacturer's instructions, except that reagent A and reagent B were both used at 1:200 dilution. The antibody binding was visualized with diaminobenzidine/hydrogen peroxide as peroxidase substrates, and the peroxidase reaction was stopped by transferring sections into $0.1 \mathrm{~mol} / \mathrm{L}$ Tris buffer $(\mathrm{pH} 8.5$ to 9). After washing, sections were mounted onto slides, air dried, and coverslipped with NeoMount (Merck, Darmstadt, Germany).

Bright-field images were taken with a Zeiss AxioImager Z1 microscope coupled to an Axiocam MRm3 digital camera, using a $20 \times$ objective. Three images $(450 \times 335 \mu \mathrm{m}$ each $)$ were taken from the cortical area of each section. To quantify microglial and astroglial cells, the percentage image area occupied by peroxidase reaction product was determined using the public domain ImageJ software version 1.45. Values obtained for images from each animal were averaged, and then used to calculate group means. All sections were processed blinded (M.B.), and codes were broken once analysis was complete.

\section{PCA and Correlation Analyses of Metabolite Profiles}

Principal component analysis (PCA), in which the coordinate axes represent the uncorrelated maximum variance directions of the data, was applied to both the standardized brain region and KA metabolome data sets, and the resulting visualizations for the first two principal components were graphed.

\section{Machine Learning}

To investigate the region specificity of the metabolite profiles, we applied two different machine learning approaches to the metabolic profiles obtained by GC-MS. First, we used a linear support vector machine classification approach $\left(\mathrm{SVM}^{19}\right)$ with default parameters to train a predictive function relating metabolite measurements to the brain region they are derived from. To estimate the predictive accuracy of the resulting model for assigning samples to the correct brain regions, we used a standard $n$-fold cross-validation scheme as follows (with two settings: $n=5$ and $n=$ total number of samples): The samples were randomly partitioned into $n$ matched-size groups, and each combination of $n-1$ of these groups was used to train the machine learning model, while the accuracy of the brain region assignment was tested for the remaining hold-out group.

To enable a visual analysis of class separability, we applied partial least squares discriminant analysis as a second machine learning algorithm $\left(\mathrm{PLS}_{-} \mathrm{DA}^{20}\right.$ ). PLS-DA is a 
statistical learning and data visualization approach designed specifically for data sets with high intercorrelation between the attributes (here: metabolites) and large numbers of attributes in relation to the numbers of samples.

Because feature selection (using only the most discriminative metabolites for the different brain regions) did not improve the cross-validated accuracy of the machine learning models for sample classification, the prediction models were built using the information from all metabolites.

\section{Statistical Analysis}

For comparison of means between controls and treated mice on neuropathology/histology or molecular marker end points, statistical analyses were done by analysis of variance, followed by the Tukey post hoc test, using the PRISM software version 5.04 (Graphpad, La Jolla, CA). $P<0.05$ was considered significant and used for interpretations. Mann-Whitney and Kolmogorov-Smirnov tests were used to confirm the comparability of metabolite profiles for the two sexes. Correlation analysis was performed with Pearson's test and two-group comparisons with unpaired $t$-test.

To obtain a ranking of the most discriminative metabolites in terms of their differential abundance across brain regions and between treatment for the KA data set (KA versus vehicle), the empirical Bayes-moderated $t$-statistic ${ }^{21}$ was used. This approach tests whether all pairwise contrasts between different outcome classes are 0 and uses an empirical Bayes method to shrink the probe-wise sample variances toward a common value.

To reduce the influence of signal-dependent noise, the variance-stabilizing transformation ${ }^{22}$ was applied to the metabolite abundance data before computing the differential abundance statistics.

For problems with more than two outcome classes (here for the comparison of different brain regions), the $F$-statistic was computed as an overall test from the $t$-statistics for every metabolite. The $P$ value significance scores were adjusted for multiple hypothesis testing. ${ }^{23}$ This allowed us to determine the set of significant metabolites as those with a false-discovery rate $<0.05$ (adjusted $P<0.05$ considered significant). Statistical analyses other than analysis of variance, $t$-test, and correlations, and all bioinformatical analyses were performed using the programming language $\mathrm{R}$ version 2.14 .2 (http://www.r-project.org).

\section{Results}

\section{The Metabolic Signature of Mouse Brain Regions}

To investigate whether the metabolite signature differed between anatomically distinct brain regions, we analyzed the metabolite profile of the following mouse brain regions by GC-MS: cortex, hippocampus, striatum, midbrain (with the main populations of dopaminergic neurons, such as substantia nigra, ventral tegmental area, and retrorubral nucleus, but excluding the colliculi), cerebellum, and brainstem (pons and medulla). We found that 191 of a total of 332 measured metabolites showed significantly differential levels across brain regions (false-discovery rate, 0.05 ); of these, 85 were identified, and 10 were derivatives of identified ones. A complete listing of the differential abundance for each of these 85 metabolites in the analyzed brain regions is shown in Supplemental Table S1.

To quantitate the differential abundance of key identified metabolites quantitatively across brain regions, we used differential abundance statistics (as described in Materials and Methods). The levels of the nine identified metabolites that showed the most differences across brain regions are shown in Figure 1. These nine metabolites were pyrophosphoric acid, taurine, 2-hydroxypyridine, phosphoethanolamine, glycine, 5oxoproline, hydrogen sulfide, dopamine, and glycerol. The differential abundance of the neurotransmitters dopamine and glycine showed an expected profile across brain regions. Dopamine and its metabolite homovanillic acid were highly abundant in the striatum, which receives the projections of dopaminergic neurons of the substantia nigra, and at least detectable in the cortex, which receives projections of dopaminergic neurons primarily from the ventral tegmental area. ${ }^{24}$ The inhibitory neurotransmitter glycine was highly abundant in the brainstem (pons and medulla), where it is known to be the major inhibitory neurotransmitter. ${ }^{25}$ Two other metabolites with a strikingly different abundance across regions were also neurotransmitters: $\gamma$-aminobutyric acid, which had the highest level in the striatum and midbrain $^{25}$; and glutamate, which had the highest level in the cortex, hippocampus, and striatum. ${ }^{9}$ These results support the robustness of our GC-MS method to analyze differentially abundant metabolites in various brain regions. The primary GC-MS data obtained for all of the metabolites that were measured are listed in Supplemental Table S2. Finally, there were also a total of 96 unknown metabolites that showed significantly differential distribution across brain regions; the most prominent of them are shown in Supplemental Figure S1. This finding points to a vast area that still awaits investigation.

To characterize and visualize the global metabolic signatures of brain regions, by taking into account all measured metabolites, we used two approaches: PCA, a classic unsupervised visualization approach for multidimensional data sets, and supervised machine learning algorithms for quantitative predictive analysis and visualization.

To generate a two-dimensional PCA plot, we choose the first two principal components that were at the basis of most of the variation between the metabolite data: $18.5 \%$ (PC1) and $12.7 \%$ (PC2), respectively. By using this approach, we obtained a first visual representation of the metabolic signatures of the different brain regions forming largely distinct clusters (Figure 2A). There was, however, some overlap between the higher brain regions, such as cortex, hippocampus, and striatum, that was possibly due to dimension reduction inherent to PCA. 

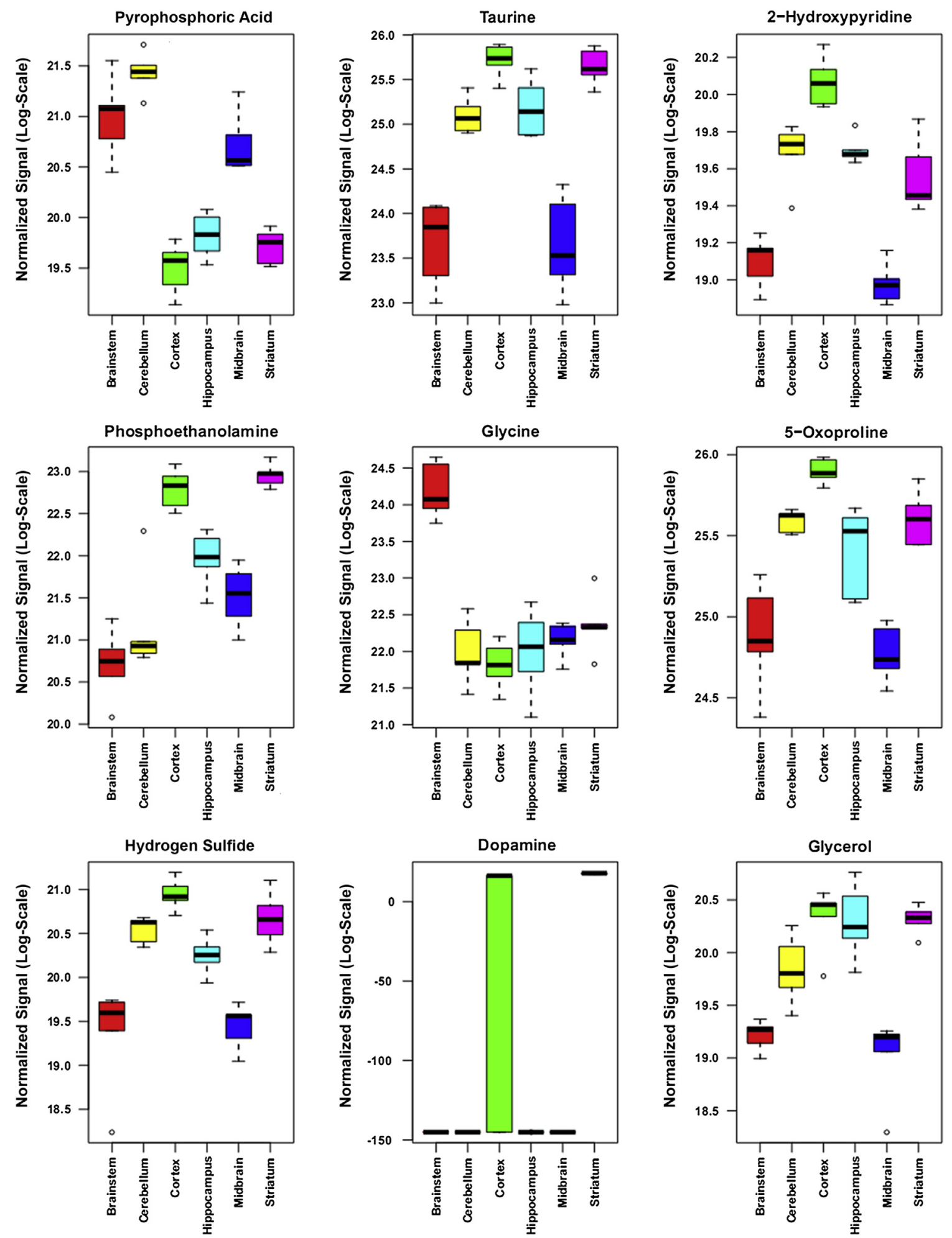

Figure 1 Box plots of nine known metabolites that were the most significantly differentially abundant across brain regions. As expected, the inhibitory neurotransmitter glycine was the most abundant in the brainstem, and the neurotransmitter dopamine was the most abundant in the striatum, and relatively high in the cortex, even though the variation was extremely high in that region. Dopamine was barely detectable in the other regions, which explains the negative log values. These observations validate the gas chromatography coupled to mass spectrometry technology used. Details are described in Results.

To investigate if the brain regions can be quantitatively differentiated on the basis of their metabolite signature, and to evaluate the utility of multidimensional brain metabolite data for predictive sample classification, we applied two different machine learning algorithms to our metabolite measurement data sets. Supervised machine learning algorithms are computer algorithms that can learn from concrete, measured data to classify objects and predict the classification of objects of 
similar nature (here, sample groups representing brain regions), and that can be used to help interpret large amounts of biological data, such as those typical for omics studies. ${ }^{26} \mathrm{We}$ used two different machine learning algorithms to classify our brain metabolite data (as described in Materials and Methods): a SVM algorithm, which separates the samples for different brain regions in high-dimensional space (with number of dimensions equal to the number of metabolites), by finding optimally separating hyperplanes; and a PLS-DA, which maps the data onto a lower-dimensional space, used for graphic visualization.

We found that, by using the first approach, SVM, the average predictive accuracy of the metabolite signature for
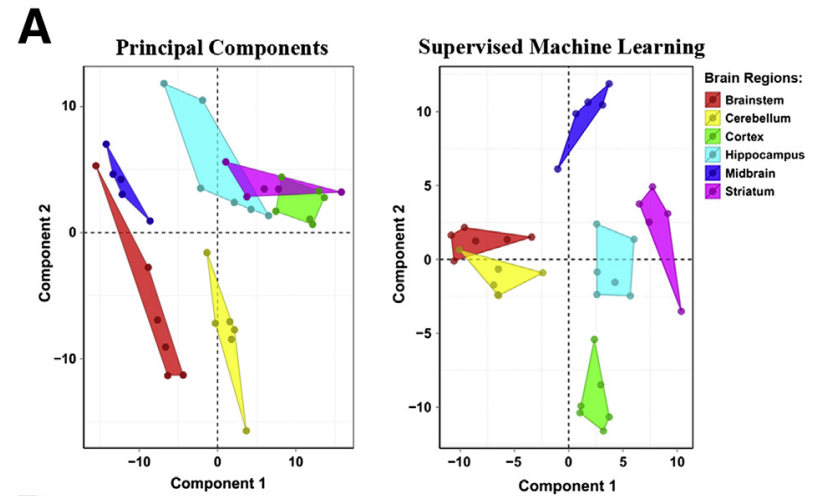

B
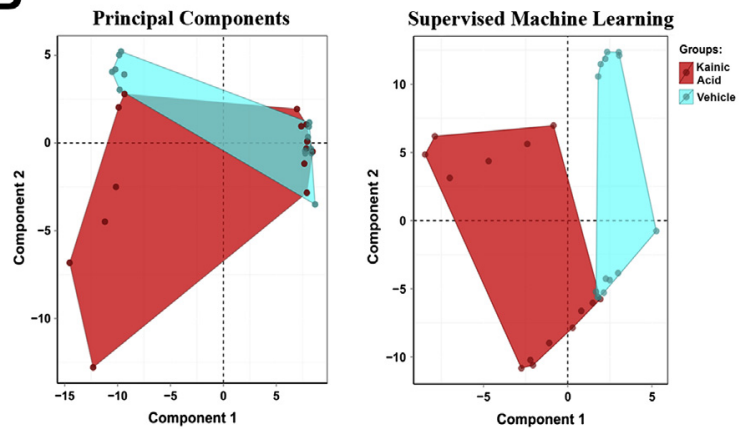

Figure 2 Visualization of metabolite profiles of different mouse brain regions $(\mathbf{A})$, and after excitotoxic brain lesioning with kainic acid (KA; B). To visualize the region-specific metabolic signatures of different mouse brain structures and of the brain's response to injury, principal component analysis (PCA) and partial least squares discriminant analysis (PLS-DA) were performed as described in Materials and Methods. A: At baseline (unmanipulated mice), the profile of different brain regions shows a clear separation. Some overlap is observed between the hippocampus, striatum, and cortex, in the PCA graph (upper left panel). The supervised machine learning algorithm PLS-DA provides a better separation, in two dimensions, between the metabolic signatures of different brain regions (upper right panel). B: After excitotoxic injury, important changes in the metabolic profile of the mouse cortex are observed. There is a significant overlap between the sample points of the two groups (vehicle versus KA) in the PCA graph (bottom left panel). A better separation between the KA- and vehicle-treated mice is achieved by using the supervised machine learning algorithm PLS-DA (bottom right panel). In this graph, three points of KA-treated groups overlap with the vehicle group, and two of three corresponded to mouse cortices that did not show signs of neurodegeneration by quantitative histology (Figure 3). Machine learning analysis of metabolite population data sets mirrors the pathological injury status of a brain sample with high accuracy, and predicts whether a given metabolite profile originates from an injured or a control brain. any of the analyzed brain regions, was between $94.29 \%$ (estimated by fivefold cross-validation) and $97.06 \%$ (estimated by leave-one-out cross-validation). To visualize, in a graph, the separability of the metabolite signature of different brain regions, we applied a second machine learning algorithm, PLS-DA. The result of this analysis, for which we used the entire data sets and obtained a training set accuracy of $97.06 \%$, is shown in Figure 2A. We observed only one misclassified sample: one cerebellum sample overlapped with the metabolite profile of the brainstem. It is unclear whether this particular sample contained a contamination, or whether PLS-DA has some limitations in separating brain regions with somewhat related metabolite profiles. Overall, though, machine learning approaches that do not use dimension reduction, in particular SVM, provided the most robust separability of brain region's metabolic profiles.

\section{The Metabolic Response to Excitotoxic Brain Injury Reflects Neurodegeneration and Gliosis}

To investigate the brain's metabolic response to a disease challenge, we used peripheral injections of KA to induce excitotoxic injury. KA, a nondegradable analog of the transmitter glutamate, is an agonist for a subtype of ionotropic glutamate receptors, and a potent epileptic and neurotoxic agent. ${ }^{27,28}$ On entering brain regions rich in glutamate receptors, such as the hippocampus and cortex, KA induces a series of cellular events leading to neurodegeneration and gliosis. ${ }^{27-29}$ Systemic injection of KA in rodent is widely used as a model to study the pathology and mechanism of neurodegenerative processes involving excitotoxicity.

We injected mice peripherally with KA or vehicle (PBS), and euthanized them 2 and 7 days after treatments. To enable detection of metabolic correlates of neurodegeneration, one hemibrain of each mouse was dissected and frozen for metabolite measurements and RNA extractions, and the other one was post fixed in paraformaldehyde for histology (as described in Materials and Methods). We focused, in this study, on the cortex, because it is a rather large region that provides sufficient amounts of material for metabolite extraction, and allows for ample and reliable sampling in histological measures. The cortex is also a region that is sensitive to excitotoxicity in mice. ${ }^{17}$

We measured the metabolic signature of the mouse cortex in KA- and vehicle-treated mice. We observed that there was no significant difference between the response to KA or vehicle at 2 and 7 days (by Mann-Whitney test to check for differences in distribution center, and by KolmogorovSmirnov to check for differences in distribution shape). This observation was confirmed by the neuropathology findings (see below), and justified combining the groups of the two treatment time points for a sufficiently powered statistical analysis.

To examine the separability of KA-induced metabolic changes versus vehicle controls, and obtain a two-dimensional visualization of the brain injury-associated multidimensional 
A
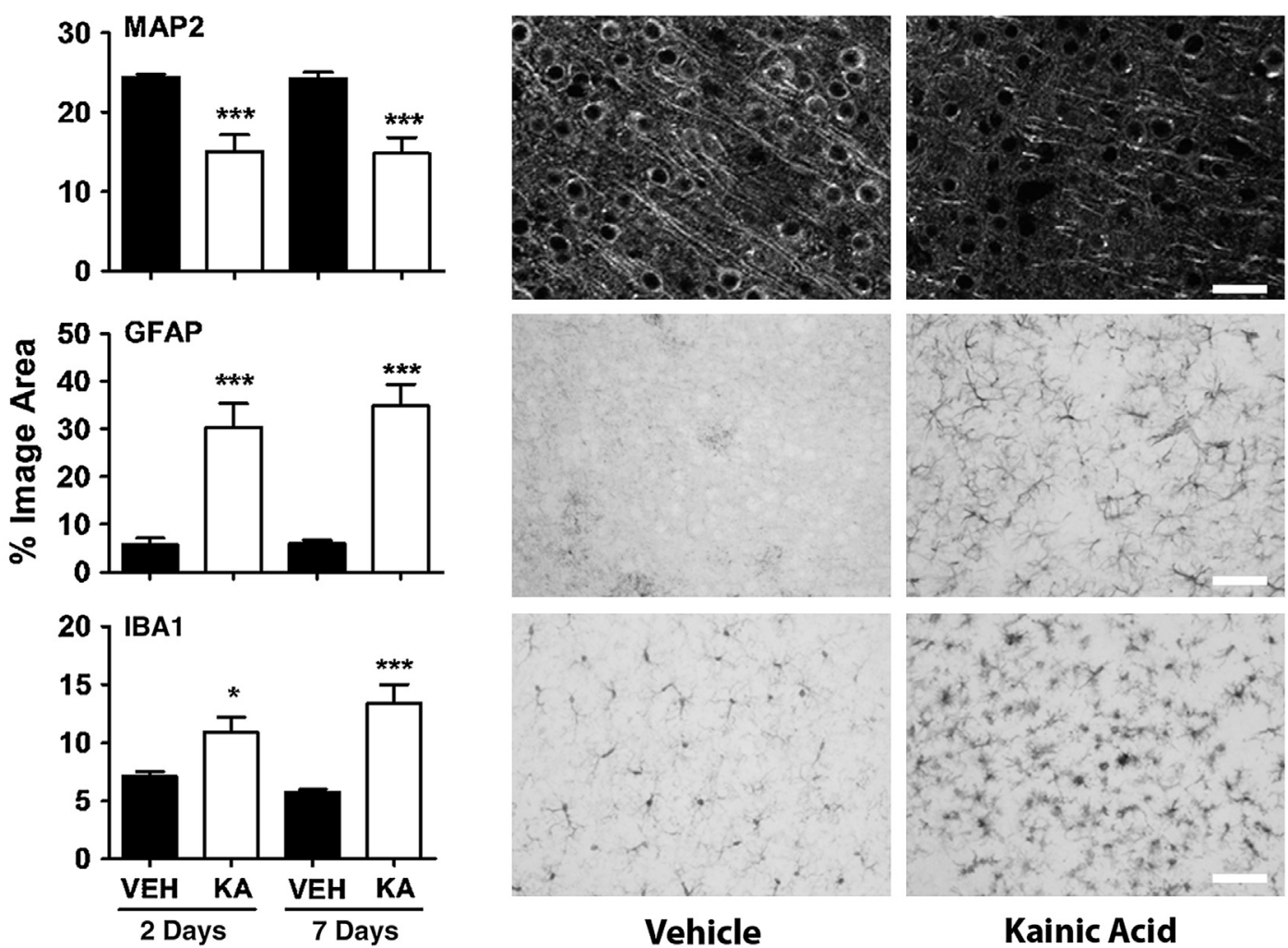

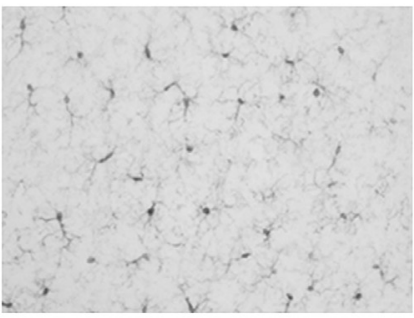

Vehicle

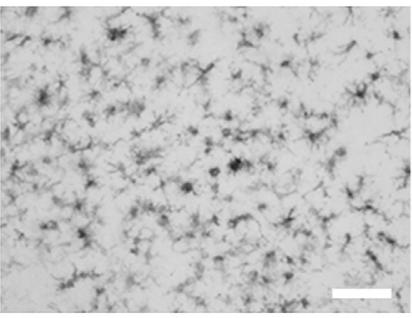

Kainic Acid

B

Astroglial Markers
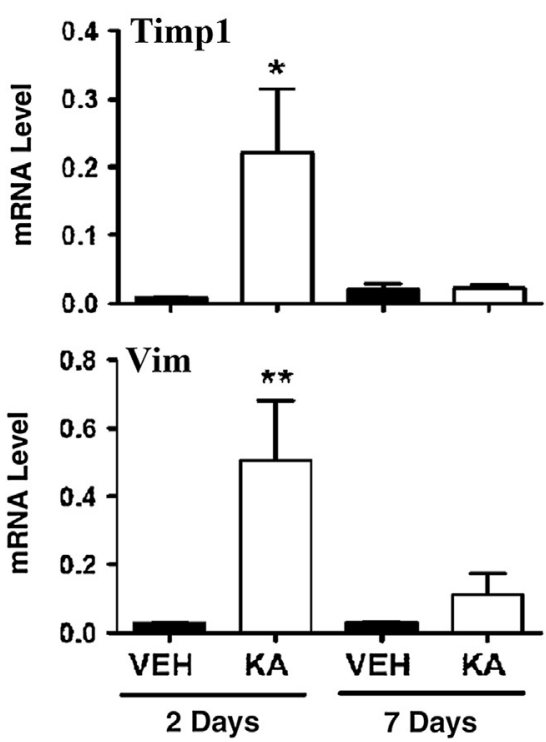

Microglial Markers
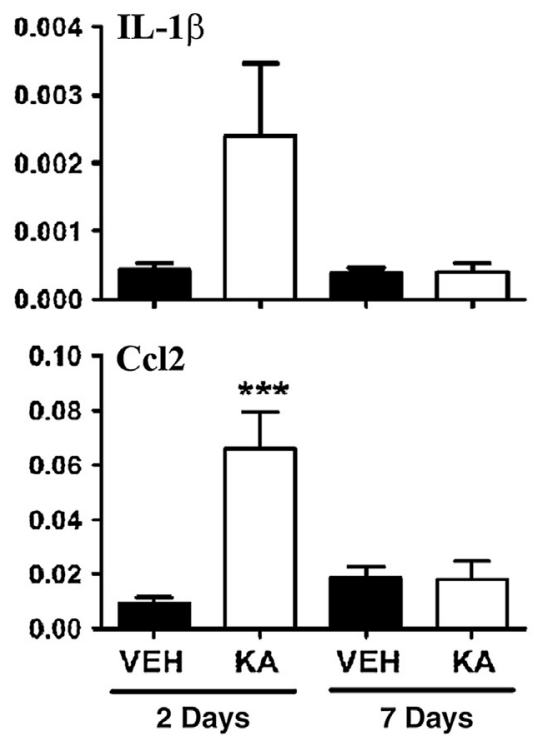

Figure 3 Neurodegeneration and gliosis induced by kainic acid (KA). Mice were injected with $20 \mathrm{mg} / \mathrm{kg} \mathrm{KA}$ or vehicle (VEH), and euthanized 2 or 7 days later. A: Histological quantitation of neurodegeneration was performed on fluorescently labeled microtubule-associated protein 2 (MAP2; neuron dendrites and cell bodies) sections. Quantitation of gliosis was performed on immunoperoxidase glial fibrillary acid protein (GFAP; reactive astrocytes) and ionizing calcium-binding adaptor molecule 1 (IBA1; microglia) labeled sections (as described in Materials and Methods). Qualitative images show examples of MAP2, GFAP, and IBA1 immunostains on VEH- or KA-treated mice. Results obtained for the synaptic markers synaptophysin were similar to those of MAP2 (Supplemental Figure S1). The extent of neurodegeneration and gliosis was similar at 2 and 7 days, probably a reflection of the high sensitivity of the FVB/N mouse strain to excitotoxicity. B: Gene expression of microglial cells and astrocyte molecular markers in KA-lesioned mouse cortex (quantitative PCR). Levels of mRNAs were measured in cortical tissues harvested and injected with VEH or $20 \mathrm{mg} / \mathrm{kg} \mathrm{KA}$ at 2 or 7 days. The levels of mRNA were determined by real-time RT-PCR and normalized using Rpl27 as housekeeping gene. Results obtained for the astroglial marker Serpina3n (not shown) were similar to those obtained for tissue metallopeptidase inhibitor 1 (Timp1) and vimentin (Vim). All markers were significant by analysis of variance, but only intergroup comparisons that showed significant increases are indicated. Data are given as means \pm SEM $(\mathbf{A}$ and $\mathbf{B}) . n=$ 6 mice (A and B). ${ }^{*} P<0.05,{ }^{*} P<0.01$, and ${ }^{* * *} P<0.001$ (A and B). Scale bars: $50 \mu \mathrm{m}$ (A, MAP2); $120 \mu \mathrm{m}$ (A, GFAP and IBA1). Ccl2, chemokine (C-C motif) ligand 2. 
metabolome data set, we first applied PCA, and then the two previously used machine learning algorithms SVM and PLSDA. The results of these analyses are shown in Figure 2B.

We observed no clear grouping pattern using PCA; the vehicle- versus KA-associated graph points showed extensive overlap (Figure 2B). Thus, PCA did not highlight any biologically relevant grouping patterns between noninjured and injured brain, and we assume that PCA-associated data dimension reduction had led to that outcome.

Therefore, to determine the separability of normal versus injured cortex metabolic signatures quantitatively and visualize it, we again applied the supervised machine learning algorithms, SVM and PLS-DA (see above). With the SVM approach, we obtained an average cross-validated accuracy of $82.7 \%$ (with fivefold cross-validation) and $82.1 \%$ (with leave-one-out cross-validation). In other words, applying supervised machine learning to the metabolite signature of mouse cortex, without any other information on its origin or treatment (injured or control mouse), nor its pathological alterations, enables the prediction of whether it originates from an injured brain with an average accuracy of $>80 \%$.

By using the second machine learning algorithm for visualization, PLS-DA, we graphed the data as shown in Figure 2B. We observed an obvious separation of the metabolic signatures of injured versus noninjured brain, with the exception that 3 of 28 graph points of KA samples were part of the overlapping region with vehicle samples, and could not be separated out.

To confirm the metabolite measurements, we set out to quantify, histologically, the extent of KA-induced neurodegeneration and gliosis after KA injection. For that purpose, we measured a set of markers: two for neuronal integrity, MAP2 and synaptophysin, which have been reported to decrease after an excitotoxic challenge, ${ }^{17}$ GFAP as a marker for astrocytosis, ${ }^{30}$ and ionizing calcium-binding adaptor molecule 1 as a marker for microgliosis. ${ }^{31}$

We observed that the extent of KA-induced neurodegeneration was $35 \%$ for MAP2 and $28 \%$ for synaptophysin at 2 and 7 days after injection (Figure 3A and Supplemental Figure S2). We also observed that the extent of KA-induced gliosis was, at both 2 and 7 days after injection, approximately twofold for microglia and fivefold for astroglia (Figure 3A). The changes induced by KA were similar at 2 and 7 days, an observation reflecting the great sensitivity of the FVB/N mouse strain to excitotoxicity. ${ }^{32}$ Thus, we combined the values of both time points for the investigation of metabolome changes induced by KA (see above). To characterize the glial cell-mediated inflammatory response to KA-induced injury, we performed a series of RNA quantitations for specific inflammatory/glial markers (as described in Materials and Methods) (Figure 3B). As expected, the expression of molecular markers of glial activity was elevated after KA administration.

Interestingly, two of three points corresponding to KAtreated mice whose metabolite profile overlapped with that of control mice (PLS-DA graph) (Figure 2B) were from mice that showed only a mitigated neurodegenerative response to
KA injection $(<5 \%$ loss of the two neuronal markers MAP2 and synaptophysin), thus being essentially noninjured. Therefore, our neuropathogical analyses showed that both machine learning algorithms, SVM and PLS-DA, provided a strong separation and, for PLS-DA, visualization of the metabolite profile of injured versus noninjured brain.

\section{Metabolic Correlates of Neurodegeneration and Gliosis}

To investigate whether specific metabolite changes correlated with the extent of neurodegeneration and/or of gliosis, and thus could provide more in-depth information about the molecular processes of excitotoxic disease process, we performed caseby-case correlation of known metabolite levels with histological and molecular marker data (as described in Materials and Methods). Perhaps not surprisingly, we observed that the decrease in one well-known correlate of neurodegeneration, $\mathrm{N}$-acetylaspartate (NAA), ${ }^{33}$ correlated significantly with the decrease in neuronal degeneration marker MAP2 $(P=0.042)$, and with the increase of astrogliosis measured by GFAP $(P=0.037)$ (Figure 4A). We detected a possibly interesting metabolic correlate of markers of astroglial reactivity, 3-hydroxybutyric acid, in mice 2 days after KA injection $(n=6)$. Its increase correlated tightly with Timpl $(P=0.008), \operatorname{Vim}(P=0.017)$, and Serpina3n $(P=0.008)$ expression increases. It was not observed 7 days after KA injection, indicating that its level is regulated. It will be interesting, in future studies, to look more closely at the regulation of the level of this metabolite after brain injury. 3-Hydroxybutyric acid is a ketone body produced primarily by hepatocytes and, in the brain, by astrocytes, ${ }^{34}$ as an alternative source of energy and neuroprotectant. ${ }^{35,36}$

We also applied a differential abundance test statistic to reveal the most prominent metabolic changes induced by excitotoxic injury. The four top-ranked known metabolites that displayed the most robust KA-induced increases were lysine, glutamine, inosine, and ribitol (median fold changes of $1.49,1.39,1.55$, and 1.18, respectively) (Figure 4B). L-Glutamine, another astroglial marker, is a metabolite of L-glutamate, whose release by neurons is enhanced after injury. ${ }^{37,38}$ Two of the other top elevated metabolites, inosine and lysine, have been reported to have neuroprotectant properties. $^{39-42}$

Thus, by investigating the brain's metabolic response to excitotoxicity, we uncovered several metabolites that, in addition to their potential use as metabolic markers for neuronal or glial response to injury, likely play an active role in the mitigation or repair of neuronal damage.

\section{Discussion}

The Metabolic Signature of Mouse Brain at Baseline and after Excitotoxic Injury

Herein, we have characterized, by using GC-MS, the metabolic signature of different mouse brain regions, and followed the 


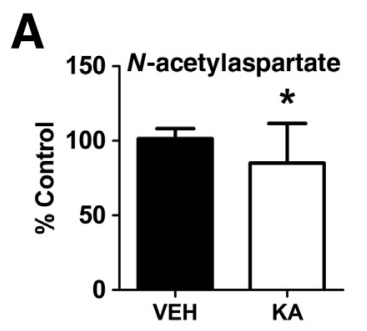

B
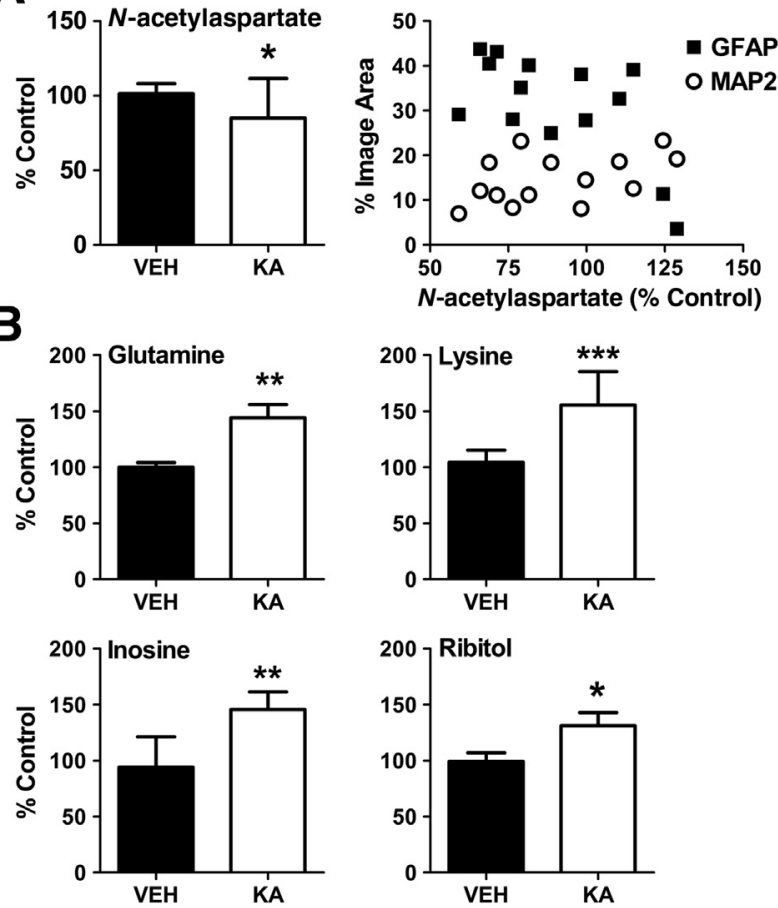

Figure 4 Metabolic correlates of excitotoxic neurodegeneration. For metabolites shown, gas chromatography coupled to mass spectrometry signal values were calculated as percentage of the means of vehicle (VEH) control. A: The neuronal metabolite $N$-acetylaspartate was significantly reduced after kainic acid (KA) injection, and correlated positively with the loss of microtubule-associated protein 2 (MAP2; $P=0.045$, Pearson $r=0.47$ ), and negatively with the increase of glial fibrillary acid protein (GFAP; $P=0.006$, Pearson $r=0.64$ ). B: Glutamine, lysine, inosine, and ribitol significantly increase after KA treatment. The increase in glutamine most likely resulted from excess glutamate released by injured neurons, which is then absorbed by astrocytes and metabolized to glutamine. The increase in lysine and inosine, which have neuroprotectant properties, may have been the brain's attempt at limiting and repairing neuronal damage. The function of ribitol is unknown. ${ }^{*} P<0.05,{ }^{*} P<0.01$, and ${ }^{* * *} P<0.001$ (unpaired $t$-test; $\mathbf{A}$ and $\mathbf{B}$ ).

changes in this signature in the mouse cortex after excitotoxic neuronal injury. We have demonstrated that, at baseline, each brain region has a distinct metabolic signature that can be best drawn out by using supervised machine learning algorithms. These algorithms provide high-accuracy tools for the predictive assessment of metabolic profiles of brain regions, and can serve as a starting point to investigate the metabolic response to different kinds of neurological disease and injury. More important, we have also demonstrated that excitotoxic neuronal injury in the mouse cortex induces a metabolic signature that reflects neuronal injury and the accompanying gliosis. Finally, we found that some metabolites that change after injury might have a role in neuronal protection and repair.

Mammalian brain regions have typically one or more unique neuronal populations and connectivity patterns. Hence, their molecular profiles and, therefore, function do not overlap. Large-scale gene expression studies have provided insights into the molecular heterogeneity of brain regions, ${ }^{43,44}$ showing, for instance, that the cerebellum had a unique gene expression signature, whereas forebrain regions tended to show more overlap. Interestingly, this is also reflected in the metabolic profile of brain regions revealed in this study: lower regions, such as the cerebellum and brainstem, had a clearly different metabolic profile than the hippocampus, cortex, and striatum, which showed some overlap.

Our study provides a map of metabolic signatures of mouse brain regions. Some known metabolites, such as dopamine or glycine, were clearly enriched in the region one would expect them to be (striatum and brainstem, respectively). Many of the detected metabolites still await identification, and it is likely that many of these metabolites have essential biological functions. For instance, we have recently identified, in activated microglial cells, the immune effectors of the brain, the metabolite itaconic acid, which has antimicrobial properties. ${ }^{15}$ We have also detected increased message levels of immunoresponsive gene 1, the mRNA coding for the enzyme catalyzing the production of that metabolite, in the brain of KA-treated mice (data not shown), indicating that it is also induced in the absence of infection.

A study on rat brain ${ }^{45}$ has analyzed the metabolic profile using ${ }^{1} \mathrm{H}$-nuclear magnetic resonance $\left({ }^{1} \mathrm{H}-\mathrm{NMR}\right)$, and has also revealed region-specific signatures, with at least some overlap to our study, in particular high levels of taurine and NAA in the cortex.

\section{The Significance of Brain Metabolic Signatures}

Mammalian brain regions, with their different neuronal subpopulations, are also characterized by differential susceptibilities to different neurodegenerative disease processes and inducing agents. ${ }^{46-53}$ Our analysis of region-specific metabolic profiles provides the basis for the investigation of how specific neurodegenerative diseases change those profiles. Excitotoxicity, for instance, a neurodegenerative process that can be experimentally induced by the administration of $\mathrm{KA}$, affects brain regions rich in glutaminergic receptors, such as hippocampus and cortex. ${ }^{17,28}$ Excitotoxic injury involves $\mathrm{Ca}^{2+}$ overload, endoplasmatic reticulum stress, disruption of cell and organelle membrane, C-junterminal kinase 3 activation, lipid peroxidation, DNA fragmentation, mitochondrial potential breakdown, ${ }^{28}$ neuroinflammation, ${ }^{27,29,54}$ and, on the basis of our findings, a specific set of metabolites that also participate in this process. Excitotoxic injury has been implicated in a range of neurological disorders, ranging from acute CNS injury and stroke to chronic afflictions, such as Alzheimer disease (AD), Huntington disease, and motor neuron disease. ${ }^{55,56}$

\section{Potential Practical Applications of Brain Metabolic Profiling in Drug and Biomarker Discovery}

Together with other end points, such as quantitative histology, metabolic profiling can be of practical use not only from a biomarker perspective, for the assessment of neuronal injury in disease models, but also to better understand the 
effect of experimental therapies and evaluate their efficacy. Because metabolic profiling can comparatively easily be scaled up and automated, it could ultimately end up being more widely used early in the lead optimization and selection process of pharmacological compounds, before more timeconsuming steps, such as quantitative histology, are used to follow up on further compound selection.

Metabolic profiling has already made its entry into the field of biomarker discovery for neurodegenerative disease. ${ }^{4}$ Many clinical studies are using NMR for brain imaging studies, or for the analysis of cerebrospinal fluid, to study metabolite changes associated with neurological diseases. ${ }^{8}$ In contrast to NMR, GC-MS provides good resolution for thermally stable compounds that can be made volatile through derivatization. ${ }^{57}$ However, although GC-MS, in contrast to NMR-based techniques, can resolve hundreds of metabolites, compound derivatization and fragmentation during the procedure prevents direct metabolite identification. Thus, GC-MS leaves several metabolites for which no standard exists unidentified. Although GC-MS has this limitation, ${ }^{1} \mathrm{H}-\mathrm{NMR}$ platforms are often restricted to a limited number of highly abundant metabolites, such as NAA, choline, creatine, or lactate, and are also, to some degree, limited in their spatial resolution. ${ }^{57}$ Thus, the precise approach to choose for metabolic profiling will depend on specific questions and needs of each study. We opted to use GC-MS, because it would allow us to capture a large population of metabolites in the mouse brain, and follow their global alterations in a disease condition.

\section{The Value of Mouse Models in Brain Metabolic Profiling}

The use of mouse models allows for longitudinal studies and the direct analysis of extracted tissue targeted by the disease or therapeutic process, thus opening the possibility of identifying and following through on biomarker candidates in a way that is not always feasible or practical in the clinic. For instance, several studies have analyzed the CNS metabolite profile of transgenic mouse models for AD.$^{58-60}$ These mice express a mutated form of the human amyloid precursor protein under the control of a neuronal promoter, and develop, as they age, several typical AD-like features, such as amyloid deposits and synaptic and neuronal degeneration and dysfunction. ${ }^{61}$ Although NAA appeared consistently decreased across these models, only one study attempted to correlate metabolite levels with histological measures, ${ }^{60}$ and showed that NAA correlated negatively with amyloid- $\beta$ plaque load. In our study, we also observed, as we had expected, a decrease in NAA in the cortex of mice after excitotoxic injury.

\section{Metabolic Correlates of Neuronal Injury as Biomarkers and Clues to Understanding the Neurodegenerative Process}

NAA, an amino acid that is present in high concentration in the brain, is a neuron-specific marker of unclear function, and a general marker for neuronal demise, in animal models and in the clinic. ${ }^{33}$ Its prominent signal in NMR studies has led to its wide use as an indicator of neuronal pathology and disease progression in a variety of CNS diseases and animal models of these diseases. ${ }^{33}$ In humans, it has been reported to be decreased in a variety of neurological afflictions, such as multiple sclerosis, AD, Parkinson disease, and Huntington disease. ${ }^{33}$ In animal models, it has been one of the most robustly affected metabolites in models of Huntington disease, ${ }^{62}$ Parkinson disease, ${ }^{63}$ and fragile- $X$ syndrome. ${ }^{64}$ Although informative as a general biomarker for neuronal demise, NAA is, diagnostically, an unspecific marker. Thus, NAA is an informative marker for monitoring neuronal damage associated with CNS diseases, even though it lacks specificity for any particular disease.

Other notable changes we have observed were metabolites that were elevated after brain injury, some of them of astroglial origin, and that are likely to play a role in neuronal protection and repair. 3-Hydroxybutyric acid was elevated 2 days after KA injection, and correlated with markers of astroglial activation. It is produced in the brain when glucose supply is limited, such as during starvation or injury. ${ }^{65}$ In vitro, it prevents neuronal damage after glucose deprivation $^{66}$ or mitochondrial toxin exposure, ${ }^{35}$ and, in vivo, it protects neurons from the effects of 1-methyl-4-phenyl1,2,3,6-tetrahydropyridine, a neurotoxin that induces Parkinson disease-like symptoms. ${ }^{36}$ Our observation that it was only elevated at a certain time point after injury indicates that its role after excitotoxicity may be restricted, and this may require further investigation in follow-up studies.

The other metabolites that were elevated were glutamine, inosine, lysine, and ribitol. Glutamine results from the conversion of neuronal glutamate after it is absorbed by astrocytes from the synaptic cleft. Under nonpathological conditions, it is returned to neurons where it is converted back into glutamate, but under pathological conditions, when neurons die, it ends up being elevated in injured tissue. $^{37,38}$ Inosine and lysine are of unknown cellular source in the injured brain, but both have been reported to have neuroprotective properties when administered after stroke. ${ }^{39-41}$ Therefore, several metabolites that are elevated after injury might reflect the attempt of surviving brain cells to limit neuronal damage and, thus, they may be biomarkers of the brain's ability to protect itself. The pathological role of the polyol sugar ribitol, which is thought to be a metabolic end product in humans, ${ }^{67}$ is unknown. Pathological accumulation of polyols might play a role in secondary neuronal damage associated with metabolic diseases, ${ }^{68,69}$ even though a direct neurotoxic effect could not be demonstrated. $^{70}$

\section{The Multidimensional Analysis of Complex Metabolite Signatures}

It is ultimately the most informative to complement individual metabolite measurements with assessing metabolite 
signatures that reflect a wider spectrum of neuronal dysfunction and degeneration, such as we have performed in this study. A population of biomarkers is far more likely than a single or even just a few molecules to capture the essence of a disease process, and to monitor the effects of therapies.

In our study, the widely used unsupervised PCA, a technique that facilitates the analysis of multidimensional data sets by providing a low-dimensional data representation and retains as much as possible of the variance information of the input data, was sufficient to separate the metabolite profile of different mouse brain regions at baseline. However, it was not optimal to distinguish between the metabolite profiles of injured and noninjured brain tissue. PCA does not necessarily preserve the distances between the original untransformed data points and, therefore, can only provide a limited visual impression of the separability between metabolite signatures. Supervised machine learning, on the other hand, turned out to be more adequate for the analysis and interpretation of metabolic profiles of brain regions and the alterations of such profiles by injury. The application of machine learning to analyze the injured mouse brain suggests that a global change in metabolic profile is an intrinsic part of the response of brain cells to injury, and that this profile could be used as a global, reliable biomarker in preclinical pharmacological efficacy studies. Machine learning, even though based on solid theoretical foundations, is still only rarely used in the assessment of large multidimensional biological data sets, and our study shows that it can be applied successfully to the study of such data sets.

Thus, we suggest that supervised machine learning algorithms can discover latent patterns in multidimensional data sets, can discriminate between different types of biological samples, and, thus, could help with the interpretation of tightly regulated and dynamic biological processes.

\section{Conclusion}

In conclusion, we have shown that, at least preclinically, a metabolic profiling approach of the mouse brain and its response to injury, guided by adequate bioinformatical evaluation and validated by quantitative neuropathology and molecular analysis, provides novel insights into brain biology and pathology. These insights could help pave the way for similar studies of biomarker candidate population identification and assessment in various models of neurodegenerative disease, and possibly their human counterparts.

\section{Acknowledgment}

We thank Dr. Karsten Hiller (Luxembourg Centre for Systems Biomedicine) for help and support with gas chromatographymass spectrometry measurements and evaluation.

\section{Supplemental Data}

Supplemental material for this article can be found at http://dx.doi.org/10.1016/j.ajpath.2015.02.016.

\section{References}

1. Sandberg R, Yasuda R, Pankratz DG, Carter TA, Del Rio JA, Wodicka L, Mayford M, Lockhart DJ, Barlow C: Regional and strainspecific gene expression mapping in the adult mouse brain. Proc Natl Acad Sci U S A 2000, 97:11038-11043

2. Benetti F, Gustincich S, Legname G: Gene expression profiling and therapeutic interventions in neurodegenerative diseases: a comprehensive study on potentiality and limits. Expert Opin Drug Discov 2012, 7:245-259

3. Di Pietro V, Amin D, Pernagallo S, Lazzarino G, Tavazzi B Vagnozzi R, Pringle A, Belli A: Transcriptomics of traumatic brain injury: gene expression and molecular pathways of different grades of insult in a rat organotypic hippocampal culture model. J Neurotrauma 2010, 27:349-359

4. Holmes E, Tsang TM, Tabrizi SJ: The application of NMRbased metabonomics in neurological disorders. NeuroRx 2006, 3:358-372

5. Jove M, Portero-Otin M, Naudi A, Ferrer I, Pamplona R: Metabolomics of human brain aging and age-related neurodegenerative diseases. J Neuropathol Exp Neurol 2014, 73:640-657

6. Kaddurah-Daouk R, Kristal BS, Weinshilboum RM: Metabolomics: a global biochemical approach to drug response and disease. Annu Rev Pharmacol Toxicol 2008, 48:653-683

7. Rochfort S: Metabolomics reviewed: a new "omics" platform technology for systems biology and implications for natural products research. J Nat Prod 2005, 68:1813-1820

8. Sinclair AJ, Viant MR, Ball AK, Burdon MA, Walker EA Stewart PM, Rauz S, Young SP: NMR-based metabolomic analysis of cerebrospinal fluid and serum in neurological diseases: a diagnostic tool? NMR Biomed 2010, 23:123-132

9. Cotman CW, Monaghan DT, Ganong AH: Excitatory amino acid neurotransmission. Annu Rev Neurosci 1988, 11:61-80

10. Hiller K, Hangebrauk J, Jager C, Spura J, Schreiber K, Schomburg D: MetaboliteDetector: comprehensive analysis tool for targeted and nontargeted GC/MS based metabolome analysis. Anal Chem 2009, 81: 3429-3439

11. Alfassi ZB: On the normalization of a mass spectrum for comparison of two spectra. J Am Soc Mass Spectrom 2004, 15:385-387

12. Hutschenreuther A, Kiontke A, Birkenmeier G, Birkenmeyer C: Comparison of extraction conditions and normalization approaches for cellular metabolomics of adherent growing cells with GC-MS Anal Methods 2012, 4:1953-1963

13. Michelucci A, Heurtaux T, Grandbarbe L, Morga E, Heuschling P: Characterization of the microglial phenotype under specific proinflammatory and anti-inflammatory conditions: effects of oligomeric and fibrillar amyloid-beta. J Neuroimmunol 2009, 210:3-12

14. Zamanian JL, Xu L, Foo LC, Nouri N, Zhou L, Giffard RG, Barres BA: Genomic analysis of reactive astrogliosis. J Neurosci 2012, 32:6391-6410

15. Michelucci A, Cordes T, Ghelfi J, Pailot A, Reiling N, Goldmann O, Binz T, Wegner A, Tallam A, Rausell A, Buttini M, Linster CL, Medina E, Balling R, Hiller K: Immune-responsive gene 1 protein links metabolism to immunity by catalyzing itaconic acid production. Proc Natl Acad Sci U S A 2013, 110:7820-7825

16. Roume H, Muller EE, Cordes T, Renaut J, Hiller K, Wilmes P: A biomolecular isolation framework for eco-systems biology. ISME J 2013, 7:110-121

17. Buttini M, Orth M, Bellosta S, Akeefe H, Pitas RE, Wyss-Coray T, Mucke L, Mahley RW: Expression of human apolipoprotein E3 or E4 
in the brains of Apoe-/- mice: isoform-specific effects on neurodegeneration. J Neurosci 1999, 19:4867-4880

18. Brionne TC, Tesseur I, Masliah E, Wyss-Coray T: Loss of TGF-beta 1 leads to increased neuronal cell death and microgliosis in mouse brain. Neuron 2003, 40:1133-1145

19. Chang C-C, Lin C-J: LIBSVM: a library for support vector machines. ACM Trans Int Sys Tech 2011, 2:27

20. Barker M, Rayens W: Partial least squares for discrimination. J Chemomet 2003, 17:166-177

21. Smyth GK: Linear models and empirical bayes methods for assessing differential expression in microarray experiments. Stat Appl Genet Mol Biol 2004, 3. Article 3

22. Huber W, von Heydebreck A, Sultmann H, Poustka A, Vingron M: Variance stabilization applied to microarray data calibration and to the quantification of differential expression. Bioinformatics 2002, 18(Suppl 1):S96-S104

23. Benjamini Y, Hochberg Y: Controlling the false discovery rate: a practical and powerful approach to multiple testing. J R Stat Soc Ser B $1995,57: 289-300$

24. Vernier P, Moret F, Callier S, Snapyan M, Wersinger C, Sidhu A: The degeneration of dopamine neurons in Parkinson's disease: insights from embryology and evolution of the mesostriatocortical system. Ann N Y Acad Sci 2004, 1035:231-249

25. Paul SM: GABA and Glycine. Psychopharmacology: The fourth generation of progress. Edited by Bloom FE, Kupfer DJ. New York, Raven Press, Ltd, 1995

26. Tarca AL, Carey VJ, Chen XW, Romero R, Draghici S: Machine learning and its applications to biology. PLoS Comput Biol 2007, 3: e116

27. Wang Q, Yu S, Simonyi A, Sun GY, Sun AY: Kainic acid-mediated excitotoxicity as a model for neurodegeneration. Mol Neurobiol 2005, 31:3-16

28. Zheng XY, Zhang HL, Luo Q, Zhu J: Kainic acid-induced neurodegenerative model: potentials and limitations. J Biomed Biotechnol 2011, 2011:457079

29. Rothman SM, Olney JW: Excitotoxicity and the NMDA receptorstill lethal after eight years. Trends Neurosci 1995, 18:57-58

30. Jacque CM, Vinner C, Kujas M, Raoul M, Racadot J, Baumann NA: Determination of glial fibrillary acidic protein (GFAP) in human brain tumors. J Neurol Sci 1978, 35:147-155

31. Ito D, Imai Y, Ohsawa K, Nakajima K, Fukuuchi Y, Kohsaka S: Microglia-specific localisation of a novel calcium binding protein, Iba1. Brain Res Mol Brain Res 1998, 57:1-9

32. Schauwecker PE, Steward O: Genetic determinants of susceptibility to excitotoxic cell death: implications for gene targeting approaches. Proc Natl Acad Sci U S A 1997, 94:4103-4108

33. Moffett JR, Ross B, Arun P, Madhavarao CN, Namboodiri AM: Nacetylaspartate in the CNS: from neurodiagnostics to neurobiology. Prog Neurobiol 2007, 81:89-131

34. Guzman M, Blazquez C: Is there an astrocyte-neuron ketone body shuttle? Trends Endocrinol Metab 2001, 12:169-173

35. Kashiwaya Y, Takeshima T, Mori N, Nakashima K, Clarke K, Veech RL: D-beta-hydroxybutyrate protects neurons in models of Alzheimer's and Parkinson's disease. Proc Natl Acad Sci U S A 2000, 97:5440-5444

36. Tieu K, Perier C, Caspersen C, Teismann P, Wu DC, Yan SD, Naini A, Vila M, Jackson-Lewis V, Ramasamy R, Przedborski S: D-betahydroxybutyrate rescues mitochondrial respiration and mitigates features of Parkinson disease. J Clin Invest 2003, 112:892-901

37. Hertz L, Zielke HR: Astrocytic control of glutamatergic activity: astrocytes as stars of the show. Trends Neurosci 2004, 27: 735-743

38. Tilleux S, Hermans E: Neuroinflammation and regulation of glial glutamate uptake in neurological disorders. J Neurosci Res 2007, 85: 2059-2070

39. Datta D, Bhinge A, Chandran V: Lysine: is it worth more? Cytotechnology 2001, 36:3-32
40. Kondoh T, Kameishi M, Mallick HN, Ono T, Torii K: Lysine and arginine reduce the effects of cerebral ischemic insults and inhibit glutamateinduced neuronal activity in rats. Front Integr Neurosci 2010, 4:18

41. Shen H, Chen GJ, Harvey BK, Bickford PC, Wang Y: Inosine reduces ischemic brain injury in rats. Stroke 2005, 36:654-659

42. Zai L, Ferrari C, Dice C, Subbaiah S, Havton LA, Coppola G, Geschwind D, Irwin N, Huebner E, Strittmatter SM, Benowitz LI: Inosine augments the effects of a Nogo receptor blocker and of environmental enrichment to restore skilled forelimb use after stroke. J Neurosci 2011, 31:5977-5988

43. Lein ES, Hawrylycz MJ, Ao N, Ayres M, Bensinger A, Bernard A, et al: Genome-wide atlas of gene expression in the adult mouse brain. Nature 2007, 445:168-176

44. Nadler JJ, Zou F, Huang H, Moy SS, Lauder J, Crawley JN, Threadgill DW, Wright FA, Magnuson TR: Large-scale gene expression differences across brain regions and inbred strains correlate with a behavioral phenotype. Genetics 2006, 174:1229-1236

45. Tsang TM, Griffin JL, Haselden J, Fish C, Holmes E: Metabolic characterization of distinct neuroanatomical regions in rats by magic angle spinning $1 \mathrm{H}$ nuclear magnetic resonance spectroscopy. Magn Reson Med 2005, 53:1018-1024

46. Calabresi P, Centonze D, Bernardi G: Cellular factors controlling neuronal vulnerability in the brain: a lesson from the striatum. Neurology 2000, 55:1249-1255

47. Kim WG, Mohney RP, Wilson B, Jeohn GH, Liu B, Hong JS: Regional difference in susceptibility to lipopolysaccharide-induced neurotoxicity in the rat brain: role of microglia. J Neurosci 2000, 20:6309-6316

48. Masliah E, Ge N, Achim CL, Hansen LA, Wiley CA: Selective neuronal vulnerability in HIV encephalitis. J Neuropathol Exp Neurol 1992, 51:585-593

49. Mattson MP, Magnus T: Ageing and neuronal vulnerability. Nat Rev Neurosci 2006, 7:278-294

50. Mitchell IJ, Griffiths MR: The differential susceptibility of specific neuronal populations: insights from Huntington's disease. IUBMB Life 2003, 55:293-298

51. Morrison BM, Hof PR, Morrison JH: Determinants of neuronal vulnerability in neurodegenerative diseases. Ann Neurol 1998, 44:S32-S44

52. Savioz A, Leuba G, Vallet PG, Walzer C: Contribution of neural networks to Alzheimer disease's progression. Brain Res Bull 2009, 80:309-314

53. Wang X, Michaelis EK: Selective neuronal vulnerability to oxidative stress in the brain. Front Aging Neurosci 2010, 2:12

54. Oprica M, Eriksson C, Schultzberg M: Inflammatory mechanisms associated with brain damage induced by kainic acid with special reference to the interleukin-1 system. J Cell Mol Med 2003, 7:127-140

55. Lau A, Tymianski M: Glutamate receptors, neurotoxicity and neurodegeneration. Pflugers Arch 2010, 460:525-542

56. Mehta A, Prabhakar M, Kumar P, Deshmukh R, Sharma PL: Excitotoxicity: bridge to various triggers in neurodegenerative disorders. Eur J Pharmacol 2013, 698:6-18

57. Urban M, Enot DP, Dallmann G, Korner L, Forcher V, Enoh P, Koal T, Keller M, Deigner HP: Complexity and pitfalls of mass spectrometry-based targeted metabolomics in brain research. Anal Biochem 2010, 406:124-131

58. Marjanska M, Curran GL, Wengenack TM, Henry PG, Bliss RL, Poduslo JF, Jack CR Jr, Ugurbil K, Garwood M: Monitoring disease progression in transgenic mouse models of Alzheimer's disease with proton magnetic resonance spectroscopy. Proc Natl Acad Sci U S A 2005, 102:11906-11910

59. Salek RM, Xia J, Innes A, Sweatman BC, Adalbert R, Randle S, McGowan E, Emson PC, Griffin JL: A metabolomic study of the CRND8 transgenic mouse model of Alzheimer's disease. Neurochem Int 2010, 56:937-947

60. von Kienlin M, Kunnecke B, Metzger F, Steiner G, Richards JG, Ozmen L, Jacobsen H, Loetscher H: Altered metabolic profile in the frontal cortex of PS2APP transgenic mice, monitored throughout their life span. Neurobiol Dis 2005, 18:32-39 
61. Games D, Buttini M, Kobayashi D, Schenk D, Seubert P: Mice as models: transgenic approaches and Alzheimer's disease. J Alzheimers Dis 2006, 9:133-149

62. Tsang TM, Woodman B, McLoughlin GA, Griffin JL, Tabrizi SJ, Bates GP, Holmes E: Metabolic characterization of the R6/2 transgenic mouse model of Huntington's disease by high-resolution MAS 1H NMR spectroscopy. J Proteome Res 2006, 5:483-492

63. Boska MD, Lewis TB, Destache CJ, Benner EJ, Nelson JA, Uberti M, Mosley RL, Gendelman HE: Quantitative $1 \mathrm{H}$ magnetic resonance spectroscopic imaging determines therapeutic immunization efficacy in an animal model of Parkinson's disease. J Neurosci 2005, 25:1691-1700

64. Davidovic L, Navratil V, Bonaccorso CM, Catania MV, Bardoni B, Dumas ME: A metabolomic and systems biology perspective on the brain of the fragile X syndrome mouse model. Genome Res 2011, 21: 2190-2202

65. Owen OE, Morgan AP, Kemp HG, Sullivan JM, Herrera MG, Cahill GF Jr: Brain metabolism during fasting. J Clin Invest 1967, 46: 1589-1595
66. Izumi Y, Ishii K, Katsuki H, Benz AM, Zorumski CF: betaHydroxybutyrate fuels synaptic function during development: histological and physiological evidence in rat hippocampal slices. J Clin Invest 1998, 101:1121-1132

67. Huck JH, Verhoeven NM, van Hagen JM, Jakobs C, van der Knaap MS: Clinical presentations of patients with polyol abnormalities. Neuropediatrics 2004, 35:167-173

68. Berry GT, Hunter JV, Wang Z, Dreha S, Mazur A, Brooks DG, Ning C, Zimmerman RA, Segal S: In vivo evidence of brain galactitol accumulation in an infant with galactosemia and encephalopathy. J Pediatr 2001, 138:260-262

69. Greene DA, Lattimer SA, Sima AA: Are disturbances of sorbitol, phosphoinositide, and $\mathrm{Na}+-\mathrm{K}+$-ATPase regulation involved in pathogenesis of diabetic neuropathy? Diabetes 1988, 37: $688-693$

70. Klusmann A, Fleischer W, Waldhaus A, Siebler M, Mayatepek E: Influence of D-arabitol and ribitol on neuronal network activity. J Inherit Metab Dis 2005, 28:1181-1183 